\title{
Study of Antibiotic Susceptibility Pattern in Staphylococcus aureus Isolated from Skin and Soft Tissue Infections in a Tertiary Care Hospital
}

\author{
Swati Rahul Dhope*, Dnyaneshwari Purushottum Ghadage, Arvind Vamanrao Bhore \\ Department of Microbiology, Smt. Kashibai Navale Medical College and \\ General Hospital, Narhe, Pune-41, India \\ *Corresponding author
}

\section{A B S T R A C T}

\section{Keywords}

Staphylococcus aureus, MRSA, Antibiotic susceptibility, Antibiotic resistance, Skin and soft tissue infections.

Article Info

Accepted:

19 June 2017

Available Online: 10 July 2017
Staphylococcus aureus is responsible for widespread infections in the community as well as hospital settings. Rapid emergence of resistance to antibiotics is well known in this organism; particularly in the Methicillin resistant Staphylococcus aureus (MRSA). So present study was carried out to detect current trend in the antibiotic susceptibility of $S$. aureus isolated from skin and soft tissue infections which in turn will be helpful for choosing empirical antibiotics for these infections. The study was conducted in our tertiary care hospital during the period of January to December 2016. S. aureus isolated from all the skin and soft tissue infections were included in the study. Antibiotics tested werepenicillin, amoxiciliin-clavulanate, cefazolin, doxycycline, erythromycin, ciprofloxacin, trimethoprim-sulfamethoxazole, vancomycin and teicoplanin. Out of $320 \mathrm{~S}$. aureus isolates, $138(43.1 \%)$ were MRSA while 182 (56.9\%) were methicillin sensitive Staphylococcus aureus (MSSA). Highest resistance was observed among ciprofloxacin $(86.6 \%)$, penicillin $(79.7 \%)$ and amoxicillin-clavulanate $(78.1 \%)$. Doxycycline and trimethoprim-sulfamethoxazole had maximum sensitivity of $86.9 \%$ and $73.8 \%$ respectively. All MRSA showed $100 \%$ sensitivity to vancomycin and teicoplanin. With this sensitivity pattern, this study emphasizes that doxycycline and trimethoprimsulfamethoxazole are the drug of choice for empirical treatment of skin and soft tissue infections caused by Staphylococcus aureus.

\section{Introduction}

Staphylococcus aureus is a ubiquitous organism responsible for widespread infections both in community as well as in hospital settings' (Tianming Li et al., 2013). These infections include pneumonia, skin and soft tissue infections, osteomyelitis, endocarditis, toxic shock syndrome etc. Skin and soft tissue infections ranged from furuncle, carbuncle, boil, impetigo, erysipelas, cellulitis, abscesses to surgical site infections etc. Incision and drainage is the primary therapy for abscesses. Along with surgical intervention, the empirical antibiotics are given for these skin and soft tissue infections. These include synthetic penicillins, first or second generation oral cephalosporins, macrolides or clindamycin. (Dennis L. Stevens et al., 2005). But staphylococcus is known for its development of rapid resistance to methicillin (MRSA). In MRSA, beta lactam antibiotics are ineffective which are most common antibiotics prescribed for the treatment of skin and soft tissue infections caused by the Staphylococcus aureus. 
With this background, it is crucial to know the antibiotic susceptibility pattern of Staphylococcus aureus in these infections for better management of patients, so present study was carried out in our hospital to find out the current trend in the antibiotic susceptibility of Staphylococcus aureus so that it can be utilized to formulate the empirical treatment for these skin and soft tissue infections.

\section{Materials and Methods}

The present study was carried out in a tertiary care hospital from January 2016 to December 2016. All specimens from skin and soft tissue infections were included in the study. All the specimens were inoculated on to blood agar and MacConkey's agar and incubated at $35^{\circ} \mathrm{C}$ for 24 hours. S. aureus was identified with standard tests such as gram stain, catalase, slide and tube coagulase etc. Antibiotic susceptibility testing was performed using standard disk diffusion method recommended by Clinical Laboratory Standards Institute (CLSI) (CLSI 2015). The following antibiotics were used- Penicillin (10U), Amoxicillin-clavulanate $(20 / 10 \mu \mathrm{g})$, Cefazolin $(30 \mu \mathrm{g})$, doxycycline $(30 \mu \mathrm{g})$, erythromycin $(15 \mu \mathrm{g})$, ciprofloxacin $(5 \mu \mathrm{g})$, trimethoprimsulfamethoxazole $\quad(1.25 / 23.75 \mu \mathrm{g})$, vancomycin $(30 \mu \mathrm{g})$ and teicoplanin $(30 \mu \mathrm{g})$. Methicillin resistance was detected by using cefoxitin $(30 \mu \mathrm{g})$ screening test as per CLSI guidelines. Vancomycin and teicoplanin were tested only for MRSA. ATCC 25923 $S$. aureus was used as quality control for interpretation of antibiotic susceptibility testing.

\section{Results and Discussion}

Total 320 S. aureus were isolated from skin and soft tissue infections. Out of 320 isolates, $182(56.9 \%)$ were Methicillin sensitive $S$. aureus (MSSA) and 138 (43.1\%) were
Methicillin resistant $S$. aureus (MRSA). Sensitivity results of different antibiotics are shown in the table 1 and chart 1.

Highest resistance was observed among ciprofloxacin $(86.6 \%)$, penicillin $(79.7 \%)$ and amoxicillin-clavulanate $(78.1 \%)$. Doxycycline and trimethoprim-sulfamethoxazole had maximum sensitivity of $86.9 \%$ and $73.8 \%$ respectively. All MRSA were $100 \%$ sensitive to vancomycin and teicoplanin.

Treatment of $S$. aureus has always remained challenging because of its rapid resistance to many antibiotics. So selection of appropriate antibiotic for $S$. aureus is essential to avoid treatment failure.

MRSA strains are rapidly increasing both in community and hospital settings. In the present study $43.1 \%$ isolates were MRSA. Similar findings were seen in the study by Indian Network for surveillance of antimicrobial resistance (INSAR) group. They found prevalence of MRSA to be $42 \%$ in 2008 and 40\% in 2009 (INSAR group, 2013).

Mild skin and soft tissue infections are treated with synthetic penicillins, first or second generation oral cephalosporins, macrolides or clindamycin (Dennis Stevens et al., 2005). This study showed that beta lactam antibiotics like penicillin, Amoxicillin-clavulanate had highest resistance, $79.7 \%$ and $78.1 \%$ respectively in MSSA. Cefazolin had resistance of $53.4 \%$. These antibiotics are ineffective in MRSA. In Onwubiko et al., study showed sensitivity of peniciilin and Amoxicillin-clavulanate to be $7.1 \%$ and $63 \%$ (Onwubiko and Sadiq et al., 2011). These findings of high resistance with beta lactam antibiotics suggest that they should not be used as empirical treatment even in MSSA.

Doxycycline followed by co-trimoxazole had highest sensitivity of $86.9 \%$ and $73.8 \%$ 
respectively in our study. These findings correlate with other studies where these antibiotics had high sensitivity (Mazhar Salim AL-Zoubi et al., 2015, Abbas et al., 2015, Huang et al., 2006). Gemmell et al., in their guidelines for prophylaxis and treatment of MRSA recommended use of tetracyclines for skin and soft tissue infections (Gemmell et al., 2006). Also both in vitro and clinical studies supported the use of doxycycline
(Ruhe and Menon et al., 2007; Ruhe et al., 2005). Review literature on clinical use of cotrimoxazole by Goldberg and Bishara recommended this drug for treatment of MRSA in skin and soft tissue infections (Goldberg and Bishara et al., 2012). Ciprofloxin had highest resistance of $86.6 \%$ in our study. Similar findings were seen by Gade et al study (Gade Qazi et al., 2013).

Table.1 Antibiotic susceptibility pattern of $S$. aureus $(\mathrm{n}=320)$

\begin{tabular}{|l|l|l|}
\hline Antibiotics & Sensitivity ( no., \%) & Resistance (no, \%) \\
\hline Penicillin & $65(20.3)$ & $255(79.7)$ \\
\hline Amoxacillin-clavulanate & $70(21.9)$ & $250(78.1)$ \\
\hline Cefazolin & $149(46.6)$ & $171(53.4)$ \\
\hline Doxycycline & $278(86.9)$ & $42(13.1)$ \\
\hline Co-trimoxazole & $236(73.8)$ & $84(26.2)$ \\
\hline Ciprofloxacin & $43(13.4)$ & $277(86.6)$ \\
\hline Erythromycin & $181(56.6)$ & $139(43.4)$ \\
\hline
\end{tabular}

Chart.1 Antibiotic sensitivity pattern of $S$. aureus

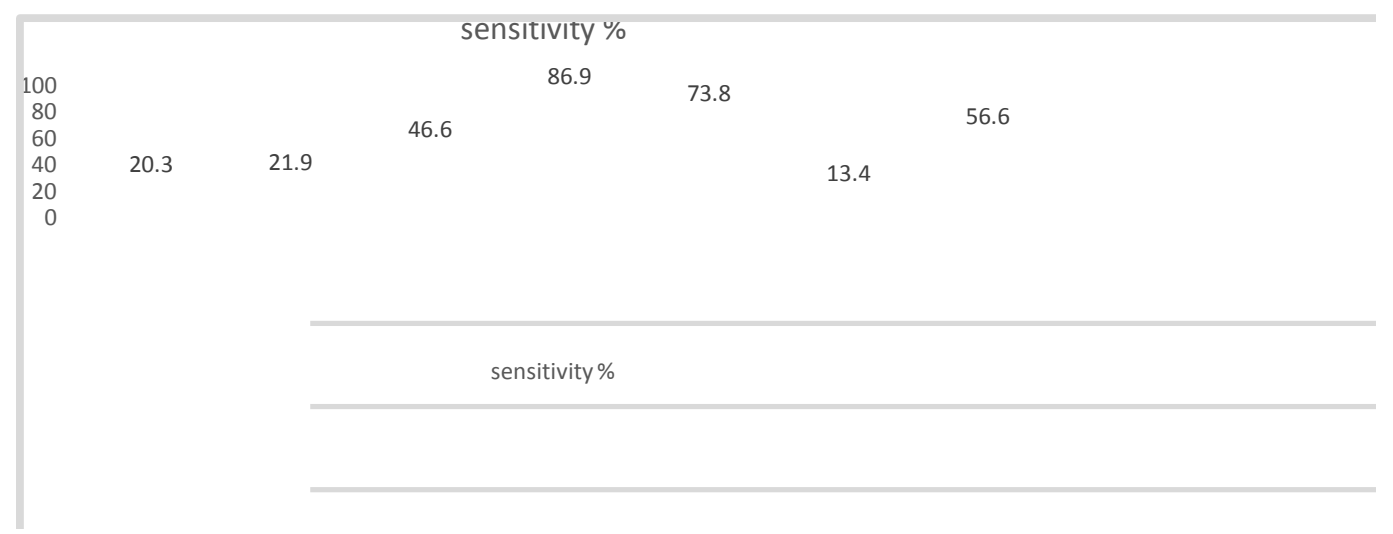

Erythromycin was $56.6 \%$ sensitive similar to other studies (Onwubiko and Sadiq et al., 2011; Mazhar Salim AL-Zoubi et al., 2015; Abbas et al., 2015). Mild skin and soft tissue infections can be managed with surgical intervention and appropriate antibiotic use. Selection of antibiotics needs regular surveillance of antibiotic susceptibility pattern. Our study suggests use of doxycycline and co-trimoxazole for mild skin and soft tissue infections on the basis of in vitro susceptibility results. But more in vivo studies are required to evaluate clinical outcome in the patients after treatment with these antibiotics. Vancomycin and linezolid should be preserved for severe skin and soft tissue infections and bacteremia.

In conclusion, mild skin and soft tissue infections caused by $S$. aureus (both MSSA and MRSA) can be empirically treated by 
doxycycline, co-trimoxazole or erythromycin. Due to high resistance of beta lactam antibiotics and ciprofloxacin, they should not be used without antibiotic susceptibility testing reports. More clinical studies are needed to evaluate clinical outcome of these drugs. Vancomycin should be used only for severe infections caused by $S$. aureus.

\section{References}

Ameer Abbas, P.S., Nirwan, Preeti Srivastava. 2015. Prevalence and antibiogram of hospital acquired-methicillin resistant Staphylococcus aureus and community acquired-methicillin resistant Staphylococcus aureus at a tertiary care hospital National Institute of Medical Sciences. Community Acquir Infect., 2: 13-5.

CLSI. 2015. Performance Standards for Antimicrobial Susceptibility Testing; Twenty -Fifth informational Supplement. CLSI document M100-S25. Wayne PA: Clinical and Laboratory Standards Institute.

Dennis, L., Stevens, et al. 2005. Practice Guidelines for the Diagnosis and Management of Skin and Soft-Tissue Infections. CID, 41: 1373-406.

Gemmell, C.G., et al. 2006. Guidelines for the prophylaxis and treatment of Methicillinresistant Staphylococcus aureus (MRSA) infections in the UK. J. Antimicrobial Chemotherapy, 57: 589-608.

Goldberg, E. and J. Bishara. 2012. Contemporary Unconventional clinical use of co-trimoxazole. Clin. Microbiol. Infect., 18(1): 8-17.

Hsin Huang, N.M., Flynn, J.H., King, Caroline Monchaud, Margaret Morita and S.H.
Cohen. 2006. Comparisons of Community-associated Methicillin resistant Staphylococcus aureus (MRSA) and Hospital-associated MRSA Infections in Sacramento, California. J. Clin. Microbiol., 44(7), pp 2423-2427.

Indian Network for surveillance of antimicrobial resistance (INSAR) group 2013. Methicillin resistant Staphylococcus aureus (MRSA) in India: Prevalence and susceptibility pattern. Indian J. Med. Res., 137, pp 363-369.

Mazhar Salim AL-Zoubi, Ibrahim Ali AlTayyar, Emad Hussein, Alla Al Jabali, SalihKhudairat. 2015. Antimicrobial susceptibility pattern of Staphylococcus aureus isolated from clinical specimens in Northern area of Jordan. Iran $J$. Microbiol., 7(5): 265-272.

Onwubiko, N.E. and N.M. Sadiq. 2011. Antibiotic sensitivity pattern of Staphylococcus aureus from tertiary health institution in Kano, Northwestern Nigeria. Pan African Med. J., 8: 4.

Ruhe, J.J., Menon, A. 2007. Tetracyclines as an oral treatment option for patients with community onset skin and soft tissue infections caused by Methicillin-resistant Staphylococcus aureus. Antimicrob. Agents Chemother., 51: 3298-3303.

Ruhe, J.J., Monson, T., Bradsher, R.W., et al. 2005. Use of long acting tetracyclines for Methicillin-resistant Staphylococcus aureus infections: case series and review of literature. Clin. Infect. Dis., 40: 14291434.

Tianming, Li, Yan Song, Yuanjun Zhu, Xin Du, Min LI. 2013. Current status of Staphylococcus aureus infection in a central teaching hospital in Shanghai, China. BMJ Microbiol., 13: 153.

\section{How to cite this article:}

Swati Rahul Dhope, Dnyaneshwari Purushottum Ghadage, Arvind Vamanrao Bhore. 2017. Study of Antibiotic Susceptibility Pattern in Staphylococcus aureus Isolated from Skin and Soft Tissue Infections in a Tertiary Care Hospital. Int.J.Curr.Microbiol.App.Sci. 6(7): 1776-1779. doi: https://doi.org/10.20546/ijcmas.2017.607.214 